\title{
Research on the Data Mining Technique and the Applications on the Modern Computer-Assisted Music Creation
}

\author{
Sui Wu \\ Hunan City University, \\ Yiyang,Hunan,413000 China
}

\begin{abstract}
In this paper, we conduct research on the data mining technique and the applications on the modern computer-assisted music creation. In today's high-speed development of modern computer technology, the computer music creation mode is widely used in the field of composing, music, recording, and other music, voice quality has become the important parts of the works, the use of the sound skills and control technology is of great significance for the feed works expressive, is better in the future study and creation of computer music will continue to explore and research fields. We use the combination of the data analysis technique to propose the novel digital music creation paradigm that will holds special significance.
\end{abstract}

Keywords- Data Mining, Applications, Computer-Assisted, Music Creation, Modern Trend.

\section{Introduction}

Music creation is a kind of art activities and art show people's feelings, also the thought of people, but not in the abstract, but with the vivid image with the main characteristics of the art lies in this. The composer at the time of conception and creation are the aesthetic experience. All creative activity, is not merely a kind of creation, also contains a kind of the feelings of anger, the creator to obtain a kind of emotional experience in the basic process of the vent. Composer's creative process is a process of emotional experience, it is stimulated by the experience of the emotion in drives the basic composer to compose. Aesthetic temperament and interest and the habitual emotions pointing directly affects the composer's inner sense of hearing, the audio works formed in the general mind, which determines the generation of the contemporary music works [1-3].

In music creation, there are a variety of aesthetic principles, contrast is one of the most common one of the most important rule. Contrast is the use of contradictory factors than the mutually lining caused by the action of strong contrast, and to highlight the theme music. In summary, the corresponding core features could be listed as the follows. (1) Using different speed in the music creation, will produce different music image, when the same composer in composing a music works, is often applied to the same music material, but in the use of the same music material, the different speed, different music image. The composer's the embodiment of the wisdom, with limited materials for a variety of basic changes, in order to achieve the different effects. (2) Adopt different timbre contrast in the music creation, music image will change timbre is one of the properties of sound, is also a factor in the form of music. Timbre due to general thinking can stimulate the audience has the capability of expression as composer on the other hand, through the timbre contrast to help shape is distinct and vivid artistic image. (3) Same theme in the creation of various composers, its characteristic will be very different musicians on the choice of the subject matter and processing, and the experiences and feelings and the thinking about things, with his unique life experience and aesthetic experience, even the same subject matter, due to differences in musicians creating personality, different method of processing subject, while its characteristics are quite different. 
Throughout the development process of music art, from the early concrete music, electronic music to the modern synthesis, to the computer music, postmodern embodies not only the change of music art form itself, more important is music aesthetic value and aesthetic spirit of change, even with the reconstruction of basic social identity that is associated with age value in delay. Computer music as a collection of music creation, music performance and music to spread, and a series of music practice in the integration of technology carrier has undoubtedly played prosperity of music art ideal huge role.

Electronic music is a recently appeared in the modern music, so by actually play to discuss about it and teaching are few. I'd like to talk through the own experience in the electronic music playing to enhance their awareness of the electronic music. For today's musicians know electronic music playing method and the potential of this new media is very important [4-5].

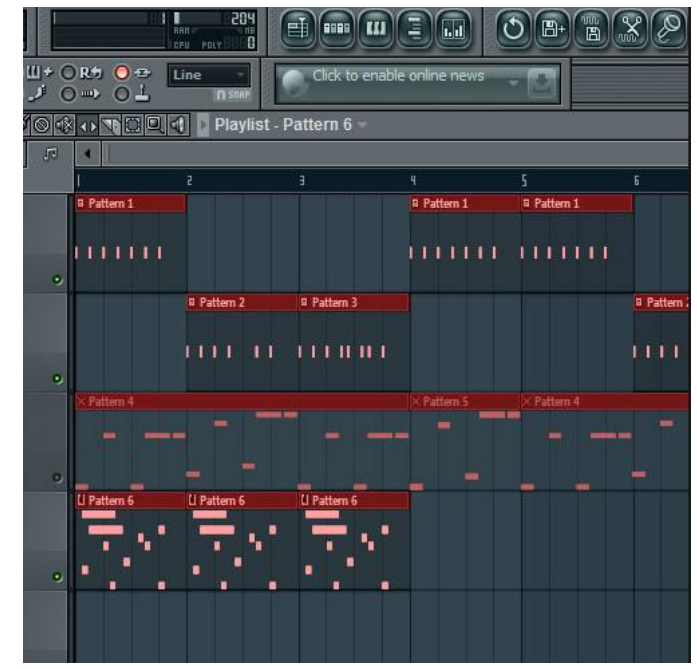

Figure 1. The Sample Interface of the Computer-Assisted Music Creation Platform

In this paper, we conduct research on the data mining technique and the applications on the modern computer-assisted music creation. When the composer to actual creation stage, the process is a whole feeling, is also a process of ultimate experience. Composers through the overall feeling are required to take full advantage of structure, choose performance means to reflect the personality of works. While the pursuit of the ultimate experience ascension the thought highly of his works to make work has the philosophy connotation. In the following sub-sections we will combine the data mining and computer to propose the novel music creation pattern to enhance the contemporary condition.

\section{The Proposed Methodology and Perspective}

The Condition of Digital Music. At present, the integration of information technology and music curriculum is information technology in higher music education in the field of application of a new and important form as is also a higher music education teaching reform and development of a kind of inevitable trend. It will completely change the traditional music teaching process and pattern, also will cause in the process of the integration of music education teaching of the profound change.

Music is an important feature of digital transmission of interactive communication. Communicator and recipient is not a one-way relationship between giving and receiving, the recipient's information feedback and communication itself together form a new form of music works, it is a creator, modify, and together create a product of the viewer, including the most direct contact between the creator and the recipient. Creators can timely understanding of the audience's attitude to his work and opinions, as the audience can accurately understand the ingenious creation intention and subtle and achieve the fulfillment of the music aesthetic information [6-7].

Digital network means the impact on the music is profound, music more open than ever under the influence of the multimedia music will thus have the following characteristics. (1) Personal composer can freely post their work in the network, as long as he is willing to, there is a certain people to access his work can be. In fact, this kind of mode of transmission directly to the audience, the creators and appreciators music 
creation, spread and appreciation as an act of pure art is full of artistic creativity and vitality. (2) Digital means not only give a person provides a very efficient means of access to the information, and more to every one may offer the best opportunity to display their talent, in the virtual digital society, offers five senses experience to stimulate technology gradually become very complete. (3) Multimedia music is a challenge to pure music forms, visual media intervention makes the viewer itself quality requirements of high pure music forms to develop in the direction of more complanation, with the deep meaning of the music is often with the help of a visual can be seen as an adjunct to the explicit expression. The emergence of multimedia culture has the very high technology support as can greatly meet the requirements of the human senses, the creators of the music creation impulse can be expressed accurately and thus the music art form is more sensitive products.
The Computer-Assisted Music Creation. Challenging music computer description of the general problem from the view of the understanding of music to the music serialization obviously is a kind of very good method, however, from the perspective of the electronic play computer only digital signal processing for the control of the music, the formal event description obviously more attractive.

Computer can only be carried out on the digital signal processing, in order to allow the computer to music signal processing, we must first to analog to digital conversion voice. Through sampling way, converting continuous music signal into the digital audio signal. Digital audio processing is based on sound theory framework, the use of digital signal processing methods and techniques the application of computer to transform and processing of digital audio, to improve or change auditory experience method. In the figure two, we show the sample interface of the music creation system.

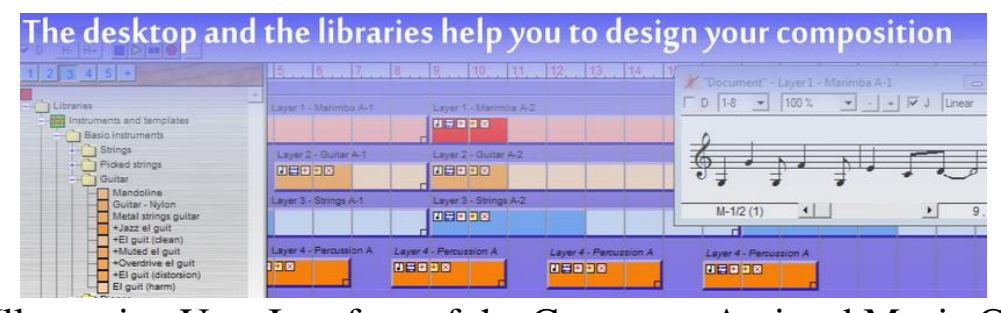

Figure 2. The Illustrative User Interface of the Computer-Assisted Music Creation System

Digital music is the technical concepts appear relatively late. Its formation process is mainly thanks to the strong function of the game market. To provide digital music, we naturally think of synthesizer, sound card, MIDI and related concepts. The computer in the field of the music intervention is deeply penetrating, infection, the transformation, including ways of the thinking, value orientation, people's spiritual world and way of life, showing obvious cultural generalization and the trend of the aesthetic generalization. In the traditional aesthetic creation, music aesthetic concepts to the creators emotional performance as the main body, emphasizes the freedom of the individual life experience, which in the part how the creator is the identity of the authority for music creation, and aesthetic conception of the computer music, more established on the basis of the interest of consumer choice [8].

Music sampling analog synthesis or synthesis of shallow depicting its scientific nature, the MIDI technology is no exception. In this approach we will answer the internal relations between different works that can be separated into the listed aspects. (1) Music in the form of study is a both difficult and controversial work. Through the axioms and rules, we can see clearly that they are in the position description and notes processing. In addition, in terms of logic system, 
they are generally not up to the mass work of music works. (2) XYZ/VERI system is an interactive computer aided certificate system, suitable for first-order temporal logic theorem proving and XYZ/SE program verification. In terms of the theorem proving, it supports the forward inference and backward inference. (3) Sound card for multimedia PC specification required by the component, is designed for a $\mathrm{PC}$ to obtain high quality acoustics and the introduction of audio interfaces. Usually equipped with a sound card, mixer, MIDI synthesizer chip interface and DSP processing unit as therefore, equipped with a sound card computer can use the corresponding software to sound recording, editing, mixing and playback function.

The Data Mining Technique. Data mining is stored in the database, data warehouse, or the other information of a large number of data in the mining process of useful knowledge. There are some terms, and data mining similar but slightly different meanings, such as database knowledge discovery, knowledge extraction, data/model analysis, archaeology, etc.

Data mining technology is conforms to the trend, it has developed the data processing technology. Data mining involves the choice of techniques and tools, the establishment of the model, including the choice of techniques and tools are very important to study, will affect the whole research process and as a result, to create a truly suitable for the mining algorithm of analysis model, data mining is the key to success. Modeling phase is the core of the data mining step at the same time as is also technical difficulty. In view of the different mining tasks, many of data mining methods are put forward. Each algorithm has its own characteristics, there are certain targeted. Different field of application of the applicable algorithms are different, even the same mining tasks, the different parts of the data for the applicability of the algorithm is also different that can be listed as the follows.
- The neural network. Neural network can be a good fit for data mining technology, it through the simulation of the human brain repeatedly learning technology to get work. For the given sample data, similar to the process of human memory neural network by learning the statistical rule of data, it can induce describe characteristics of sample data model, then using the data model we have learned to classification of new data is given.

- The decision tree method. This is a commonly used method as it can be used for data analysis or prediction. The decision tree classification or decision tree said to the collection, and then through the data set of classification rules and law of development. Using mutual information in information theory (information gain) find in database, a field that has the largest amount of information to build a decision tree node, then according to the different values of the field to establish branches of the tree [9].

- The rough set method. The theory is not to need to advance the characteristics of the given number of the certain characteristics or attributes describe, but directly from a given problem, through the indiscernibility relation and approximate indiscernibility classes to determine the problem domain, so as to find out the inherent law in the question while this method plays an important role in data mining and is often used to deal with vagueness and uncertainty.

The Enhanced Musical Creation Paradigm. Music creators in the subjective dynamic role in the process of thesis summarized the starting point of the first performance for clear art. There are two different starting points which made the common properties of the general matters as the starting point, essentially abstract concept as the starting point, and then seek individual image, to 
input the "essence", make individual general images. An individual image is the individual things, as a starting point, to find and feel some novel and unique factors, and then within the scope of the wider social life, to confirm and test the findings and feelings, and together, as a complete artistic image, which shows some essential aspects of social and historical phenomenon.

In computer technology and consumer culture, under the function of music art also like other kinds of art activities presents the obvious aesthetic generalization tendency, any the experience of everyday life can become the aesthetic experience, any natural sound can be to take advantage of aesthetics, any music creation can be directly to the audience orientation of the basic senses, anyone can become the aesthetic object of music works, any music works can have unlimited copies for the public to enjoy, so on. Although the electronic music history is short, the lack of the traditional, as a traditional trained violinist play electronic music, we feel a duty with serious attitude and trained ear for studying the electronic music. Electronic music along with the rapid development of the science and technology development, we hope that with the continuous development of science, the problems existing in the electronic music playing are constantly exposed.

Electronic music play of my experience told me that a performer for the audience to hear all the sounds, even to the electronic noise is not controlled by the player directly responsible. As much as possible in order to express their art, any one player before the audience heard the idea of playing is responsible for the sound research. Similarly, no one wants to work in can see effect was performed before the premiere of the composer, all must consider the environmental factors in the influence of the real show. These responsibilities are not only suitable for those who use electronic instruments and more specifically they fell on the shoulders of those who think of the future for performing arts.

\section{Conclusion}

In this paper, we conduct research on the data mining technique and the applications on the modern computer-assisted music creation. Computer music thinking involves two aspects: the influence of the computer itself tool characteristics and the concept of the composer's music. It is worth noting, in computer music, the formation of many thinking features not only since the computer used in the music only, but long before computers were initiation and has been widely accepted. Therefore, the computer music "prehistory" recalled some of the significant phenomenon, ideas and thinking can make us on the origin and development of computer music thinking have a more clear understanding. Under this background, we propose the data mining technique and the applications on the modern computer-assisted music creation to serve as the novel research on the related areas.

\section{References}

[1] Corrêa, Ana Grasielle Dionísio, et al. "Computer assisted music therapy: A case study of an augmented reality musical system for children with cerebral palsy rehabilitation." Advanced Learning Technologies, 2009. ICALT 2009. Ninth IEEE International Conference on. IEEE, 2009.

[2] McKay, Cory, and Ichiro Fujinaga. "Style-independent computer-assisted exploratory analysis of large music collections." Journal of Interdisciplinary Music Studies 1.1 (2007): 63-85.

[3] Farbood, Morwaread, et al. "System and Method for Music Creation and Distribution Over Communications Network." U.S. Patent Application No. 11/456,739.

[4] Allaway, Heather C., et al. "Digit ratios (2D: 4D) determined by computer - assisted 
analysis are more reliable than those using physical measurements, photocopies, and printed scans." American Journal of Human Biology 21.3 (2009): 365-370.

[5] Seddon, Frederick A. "Collaborative computer-mediated music composition in cyberspace." British Journal of Music Education 23.03 (2006): 273-283.

[6] Huang, Michael Xuelin, et al. "MelodicBrush: a novel system for cross-modal digital art creation linking calligraphy and music." Proceedings of the Designing Interactive Systems Conference. ACM.
[7] Tjora, Aksel H. "The groove in the box: a technologically mediated inspiration in electronic dance music." Popular Music 28.02 (2009): 161-177.

[8] McLoughlin, Catherine, and Joe Luca. Applying situated learning theory to the creation of learning environments to enhance socialisation and self-regulation. na, 2006.

[9] Dal Farra, Ricardo. "Something lost, something hidden, something found: electroacoustic music by Latin American composers." Organised Sound 11.2 (2006): 131-142. 